\title{
OPTIMAL CONTROL OF A LARGE DAM
}

\author{
VYACHESLAV M. ABRAMOV,${ }^{*}$ Monash University
}

\begin{abstract}
A large dam model is the object of study of this paper. The parameters $L^{\text {lower }}$ and $L^{\text {upper }}$ define its lower and upper levels, $L=L^{\text {upper }}-L^{\text {lower }}$ is large, and if the current level of water is between these bounds, the dam is assumed to be in a normal state. Passage across one or other of the levels leads to damage. Let $J_{1}$ and $J_{2}$ denote the damage costs of crossing the lower and, respectively, the upper levels. It is assumed that the input stream of water is described by a Poisson process, while the output stream is state dependent. Let $L_{t}$ denote the dam level at time $t$, and let $p_{1}=\lim _{t \rightarrow \infty} \mathrm{P}\left\{L_{t}=L^{\text {lower }}\right\}$ and $p_{2}=\lim _{t \rightarrow \infty} \mathrm{P}\left\{L_{t}>L^{\text {upper }}\right\}$ exist. The long-run average cost, $J=p_{1} J_{1}+p_{2} J_{2}$, is a performance measure. The aim of the paper is to choose the parameter controlling the output stream so as to minimize $J$.
\end{abstract}

Keywords: Dam; state-dependent queue; asymptotic analysis; control problem

2000 Mathematics Subject Classification: Primary 60K30; 60K25

Secondary 40E05; 90B05

\section{Introduction}

A large dam model is the object of study of this paper. The parameters $L^{\text {lower }}$ and $L^{\text {upper }}$ are respectively the lower and upper levels of the dam, and if the current level of water is between these bounds, the dam is assumed to be in a normal state. The reason for calling the dam large is that the difference $L=L^{\text {upper }}-L^{\text {lower }}$ is large. Assuming this property of the dam enables us to use asymptotic analysis (as $L \rightarrow \infty$ ) and obtain much simpler representations for the desired characteristics of the model than we would were the dam not large. In turn, these representations are then easily used to solve the appropriate control problems formulated below.

In the literature, dam, storage, and production models are associated with state-dependent queueing systems (see, e.g. [1], [2], [7]-[10], [12]-[14], [21], and others). The model of the present paper is the following. We assume that units of water arriving at the dam are registered by counter at random instants $t_{1}, t_{2}, \ldots$, and that the interarrival times, $\tau_{n}=t_{n+1}-t_{n}$, are mutually independent, exponentially distributed random variables with parameter $\lambda$. Outflow of water is state dependent in the following sense. If the level of water is between $L^{\text {lower }}$ and $L^{\text {upper }}$, then the duration of an interval between unit departures has the probability distribution $B_{1}(x)$. If the level of water increases above the level $L^{\text {upper }}$, then the probability distribution of the duration of an interval between unit departures is $B_{2}(x)$. It is also assumed that if the level of water is exactly $L^{\text {lower }}$, then the departure process of water is frozen, and is resumed as soon as the level of water exceeds the value $L^{\text {lower }}$. It is worth noting that policies according to which changes in the service rate depend on the dam level are of importance in the literature (see [1], [2], [7], [8], [21], and others). However, all of them involve performance measures

Received 21 December 2005; revision received 17 November 2006.

* Postal address: School of Mathematical Sciences, Monash University, Building 28M, Clayton Campus, Clayton, VIC 3800, Australia. Email address: vyacheslav.abramov@sci.monash.edu.au 
associated with an appropriate upper level of water in a dam, and to the author's knowledge the known results on the performance analysis of river flows structured by both lower and upper levels are difficult to derive and hard to apply to real-world situations even for simple models (see, e.g. the review paper [15]). Furthermore, in most studies the explicit representations are in terms of the Laplace-Stieltjes transforms of the initial distributions, and there is no literature on the asymptotic analysis of large dams.

In terms of queueing theory, the problem considered in this paper can be reformulated as follows. Consider a single-server queueing system where the arrival flow of customers is Poisson with rate $\lambda$ and the service time of a customer depends upon queue length as follows. If, at the moment the customer's service begins, the number of customers in the system is not greater than $L$, then the service time of this customer has the probability distribution $B_{1}(x)$. Otherwise (if there are more than $L$ customers in the system at the moment the customer's service begins), the probability distribution of the service time of this customer is $B_{2}(x)$. The analytical results for this queueing system are known (see, e.g. [3, Chapter 2]). Notice that the lower level of the dam, $L^{\text {lower }}$, corresponds to zero queue length. The dam specification of the problem is characterized by performance criteria which, in terms of the queueing formulation, are as follows. Let $q_{t}$ denote the queue length at time $t$. The problem is to choose the output parameter of the system so as to minimize the functional $J(L)=p_{1}(L) J_{1}(L)+p_{2}(L) J_{2}(L)$, where $p_{1}(L)=\lim _{t \rightarrow \infty} \mathrm{P}\left\{q_{t}=0\right\}, p_{2}(L)=\lim _{t \rightarrow \infty} \mathrm{P}\left\{q_{t}>L\right\}$, and $J_{1}(L)$ and $J_{2}(L)$ are the corresponding damage costs, proportional to $L$. To be precise, assume that $J_{1}(L)=j_{1} L$ and $J_{2}(L)=j_{2} L$, where $j_{1}$ and $j_{2}$ are positive constants. Assuming that $L \rightarrow \infty$, we shall often write $p_{1}$ and $p_{2}$ (without the argument $L$ ) rather than $p_{1}(L)$ and $p_{2}(L)$. The argument $L$ will be often omitted in other functions; in particular, we shall freely write $J, J_{1}$, and $J_{2}$ without it.

To specify the problem more correctly, we assume that the input parameter $\lambda$ and the probability distribution function $B_{2}(x)$ are given, while $B_{1}(x) \equiv B_{1}(x, C)$ is a family of probability distributions depending on the parameter $C \geq 0$, which in turn is closely related to the expectation $\int_{0}^{\infty} x \mathrm{~d} B_{1}(x)$. Then the output rate associated with the probability distribution $B_{1}(x)$ can be changed so that the minimum value of the functional is associated with the choice of the parameter $C$, resulting in the choice of the function $B_{1}(x, C)$. A verification of the correctness of this formulation and a more concrete description of the parameter $C$ will be given in the sequel (see the formulations of Theorems 4.1, 4.2, 4.3, and 4.4). It is interesting to note that the solution to the above control problem is asymptotically independent of the explicit form of the probability distribution functions $B_{1}(x)$ and $B_{2}(x)$, and depends only on the expectations $\int_{0}^{\infty} x \mathrm{~d} B_{2}(x)<1 / \lambda$ and $\int_{0}^{\infty} x \mathrm{~d} B_{1}(x)$ and the integral $\int_{0}^{\infty} x^{2} \mathrm{~d} B_{1}(x)$. The details of this dependence will be explained later.

We use the notation $b_{i}=\int_{0}^{\infty} x \mathrm{~d} B_{i}(x)$ and $\rho_{i}=\lambda b_{i}$, for $i=1,2$, and assume that $\rho_{2}<1$. This assumption is a standard condition for stationarity, ergodicity of the queue length process $q_{t}$, and existence of the limits $p_{1}$ and $p_{2}$ (independent of the initial state of the process). In addition to this, we shall also assume the existence of the third moment: $\rho_{1, k}=\lambda^{k} \int_{0}^{\infty} x^{k} \mathrm{~d} B_{1}(x)<\infty, k=2,3$. The existence of the second moment is used in Theorem 3.1, and the existence of the third moment of each member of the specified family of distributions $B_{1}(x, C)$ is required in Theorems 4.1, 4.2, 4.3, and 4.4.

The special features of the present paper are as follows.

- We solve the control problem in which the performance criteria take into account passage across the upper and lower levels. The formulation of the problem is not traditional, but realistic. Other, similar control problems arising in practice can be solved by adapting the method of this paper. 
- The presentation of the results is clear and makes them amenable to real applications.

- The mathematical methods of the paper are not traditional, but are clear and easily understandable.

The paper is structured as follows. In Section 2 we discuss the state-dependent queue length process and derive representations for the probabilities $p_{1}$ and $p_{2}$. Section 3 contains the results on the asymptotic analysis of $p_{1}$ and $p_{2}$. The main result on this asymptotic behaviour is given in Theorem 3.1. In Section 4 some additional theorems on the asymptotic behaviour of $p_{1}$ and $p_{2}$ are proved and then used to solve the control problem. The main result of the paper, solution of the control problem, is formulated in Section 5. Concluding remarks are given in Section 6.

\section{The state-dependent queue and its characteristics in a busy period}

In this section we discuss the main characteristics of the state-dependent queueing system described in the introduction. Let $T_{L}, I_{L}$, and $v_{L}$ respectively denote the duration of a busy period, the duration of an idle period, and the number of customers served during a busy period. Let $T_{L}^{(1)}$ and $T_{L}^{(2)}$ respectively denote the total times during a busy period when $0<q_{t} \leq L$ and $q_{t}>L$, and let $v_{L}^{(1)}$ and $v_{L}^{(2)}$ respectively denote the total numbers of customers served during a busy period when $0<q_{t} \leq L$ and $q_{t}>L$. We have the following two obvious equations:

$$
\begin{aligned}
& \mathrm{E} T_{L}=\mathrm{E} T_{L}^{(1)}+\mathrm{E} T_{L}^{(2)}, \\
& \mathrm{E} v_{L}=\mathrm{E} v_{L}^{(1)}+\mathrm{E} v_{L}^{(2)} .
\end{aligned}
$$

According to Wald's equation (see [11, p. 384]),

$$
\begin{aligned}
& \mathrm{E} T_{L}^{(1)}=b_{1} \mathrm{E} v_{L}^{(1)}, \\
& \mathrm{E} T_{L}^{(2)}=b_{2} \mathrm{E} v_{L}^{(2)} .
\end{aligned}
$$

Now, the number of arrivals during a busy cycle coincides with the total number of customers served during a busy period. Hence, by applying Wald's equation again and taking into account (2.1)-(2.4), we obtain

$$
\begin{aligned}
\lambda \mathrm{E} T_{L}+\lambda \mathrm{E} I_{L} & =\lambda \mathrm{E} T_{L}+1 \\
& =\lambda \mathrm{E} T_{L}^{(1)}+\lambda \mathrm{E} T_{L}^{(2)}+1 \\
& =\rho_{1} \mathrm{E} v_{L}^{(1)}+\rho_{2} \mathrm{E} v_{L}^{(2)}+1 \\
& =\mathrm{E} v_{L}^{(1)}+\mathrm{E} v_{L}^{(2)} .
\end{aligned}
$$

From (2.5) we have

$$
\mathrm{E} v_{L}^{(2)}=\frac{1}{1-\rho_{2}}-\frac{1-\rho_{1}}{1-\rho_{2}} \mathrm{E} v_{L}^{(1)},
$$

expressing $\mathrm{E} v_{L}^{(2)}$ in terms of $\mathrm{E} v_{L}^{(1)}$. For example, if $\rho_{1}=1$ then $\mathrm{E} v_{L}^{(2)}=1 /\left(1-\rho_{2}\right)$ for any $L$. A similar equation holds for $\mathrm{E} T_{L}^{(2)}$ : from (2.4) and (2.6) we obtain

$$
\mathrm{E} T_{L}^{(2)}=\frac{\rho_{2}}{\lambda\left(1-\rho_{2}\right)}-\frac{\rho_{2}\left(1-\rho_{1}\right)}{\lambda\left(1-\rho_{2}\right)} \mathrm{E} T_{L}^{(1)} .
$$


Equations (2.6) and (2.7) enable us to obtain the stationary probabilities $p_{1}$ and $p_{2}$. By applying first the renewal reward theorem (see, e.g. [17, p. 78]) and then (2.5) and (2.6), for $p_{1}$ we obtain

$$
p_{1}=\frac{\mathrm{E} I}{\mathrm{E} T_{L}^{(1)}+\mathrm{E} T_{L}^{(2)}+\mathrm{E} I}=\frac{1}{\mathrm{E} v_{L}^{(1)}+\mathrm{E} v_{L}^{(2)}}=\frac{1-\rho_{2}}{1+\left(\rho_{1}-\rho_{2}\right) \mathrm{E} v_{L}^{(1)}} .
$$

Analogously,

$$
p_{2}=\frac{\mathrm{E} T_{L}^{(2)}}{\mathrm{E} T_{L}^{(1)}+\mathrm{E} T_{L}^{(2)}+\mathrm{E} I}=\frac{\rho_{2} \mathrm{E} v_{L}^{(2)}}{\mathrm{E} v_{L}^{(1)}+\mathrm{E} v_{L}^{(2)}}=\frac{\rho_{2}+\rho_{2}\left(\rho_{1}-1\right) \mathrm{E} v_{L}^{(1)}}{1+\left(\rho_{1}-\rho_{2}\right) \mathrm{E} v_{L}^{(1)}} .
$$

\section{Asymptotic analysis of $p_{1}$ and $p_{2}$ as $L$ increases to infinity}

By sample path analysis and the property of the lack of memory of exponential distributions, it follows that the random variable $v_{L}^{(1)}$ coincides in distribution with the number of customers served during a busy period of the M/GI/1/L queueing system (the parameter $L$ denotes the number of customers in the system excluding the customer at the server). Specifically, we use the fact that during a busy period the number of times service begins when the number of customers in the system does not exceed $L$ coincides with the number of arrivals when the number of customers in the system does not exceed $L+1$. We also use the fact that the residual interarrival time after a service completion has an exponential distribution with parameter $\lambda$.

Therefore, the known results for M/GI/1/L queueing systems can be used. It is known (see, e.g. [3] and [4]) that $E v_{L}^{(1)}$ is determined by the convolution-type recurrence relation

$$
\mathrm{E} v_{L}^{(1)}=\sum_{j=0}^{L} \mathrm{E} v_{L-j+1}^{(1)} \int_{0}^{\infty} \mathrm{e}^{-\lambda x} \frac{(\lambda x)^{j}}{j !} \mathrm{d} B_{1}(x), \quad \mathrm{E} v_{0}^{(1)}=1,
$$

where $\mathrm{E} v_{n}^{(1)}, n=1,2, \ldots$, denotes the expectation of the number of customers served during a busy period in an $\mathrm{M} / \mathrm{GI} / 1 / n$ queue.

The probabilities $p_{1}(L)$ and $p_{2}(L)$ are expressed explicitly in terms of $\mathrm{E} v_{L}^{(1)}$, and their asymptotic behaviours (as $L \rightarrow \infty$ ) can be obtained from the following known results. Let $Q_{0}>0$ be an arbitrary real number and, for $n \geq 0$, let

$$
Q_{n}=\sum_{j=0}^{n} r_{j} Q_{n-j+1}
$$

where $r_{0}>0, r_{j} \geq 0$, and $r_{0}+r_{1}+\cdots=1$. Let $r(z)=\sum_{j=0}^{\infty} r_{j} z^{j},|z| \leq 1$, be a generating function and let $\gamma_{m}=\lim _{z \uparrow 1} r^{(m)}(z)$, where $r^{(m)}(z)$ is the $m$ th derivative of $r(z)$. Notice that the sequence $\left\{Q_{n}\right\}$ is increasing and that

$$
\sum_{n=0}^{\infty} Q_{n} z^{n}=\frac{Q_{0} r(z)}{z-r(z)}
$$

(see [16, Section 25] and [20, pp. 22-23]).

Lemma 3.1. ([20, pp. 22-23].) If $\gamma_{1}<1$ then

$$
\lim _{n \rightarrow \infty} Q_{n}=\frac{Q_{0}}{1-\gamma_{1}}
$$


If $\gamma_{1}=1$ and $\gamma_{2}<\infty$ then

$$
\lim _{n \rightarrow \infty} \frac{Q_{n}}{n}=\frac{2 Q_{0}}{\gamma_{2}}
$$

If $\gamma_{1}>1$ then

$$
\lim _{n \rightarrow \infty}\left[Q_{n}-\frac{Q_{0}}{\sigma^{n}\left(1-r^{\prime}(\sigma)\right)}\right]=\frac{1}{1-\gamma_{1}},
$$

where $\sigma$ is the root of the functional equation $z=r(z)$ that is least in absolute value.

From this lemma we can derive the asymptotic behaviours of the probabilities $p_{1}$ and $p_{2}$. For $\operatorname{Re}(s) \geq 0$, denote by $\hat{B}_{1}(s)$ the Laplace-Stieltjes transform of $B_{1}(x)$. We have the following theorem.

Theorem 3.1. If $\rho_{1}<1$ then

$$
\begin{aligned}
& \lim _{L \rightarrow \infty} p_{1}(L)=1-\rho_{1}, \\
& \lim _{L \rightarrow \infty} p_{2}(L)=0 .
\end{aligned}
$$

If $\rho_{1}=1$ then

$$
\begin{aligned}
& \lim _{L \rightarrow \infty} L p_{1}(L)=\frac{\rho_{1,2}}{2}, \\
& \lim _{L \rightarrow \infty} L p_{2}(L)=\frac{\rho_{2}}{1-\rho_{2}} \frac{\rho_{1,2}}{2} .
\end{aligned}
$$

If $\rho_{1}>1$ then

$$
\lim _{L \rightarrow \infty} \frac{p_{1}(L)}{\varphi^{L}}=\frac{\left(1-\rho_{2}\right)\left[1+\lambda \hat{B}_{1}^{\prime}(\lambda-\lambda \varphi)\right]}{\rho_{1}-\rho_{2}},
$$

where $\varphi$ is the root of the functional equation $z=\hat{B}_{1}(\lambda-\lambda z)$ that is least in absolute value, and

$$
\lim _{L \rightarrow \infty} p_{2}(L)=\frac{\rho_{2}\left(\rho_{1}-1\right)}{\rho_{1}-\rho_{2}} .
$$

Proof. The proof of this theorem follows by application of Lemma 3.1. Straightforward application of the aforementioned lemma to the recurrence relation for $\mathrm{E} v_{L}^{(1)}$ yields the following. If $\rho_{1}<1$ then

$$
\lim _{L \rightarrow \infty} E v_{L}^{(1)}=\frac{1}{1-\rho_{1}} .
$$

If $\rho_{1}=1$ then

$$
\lim _{L \rightarrow \infty} \frac{E v_{L}^{(1)}}{L}=\frac{2}{\rho_{1,2}}
$$

If $\rho_{1}>1$ then

$$
\lim _{L \rightarrow \infty}\left[E v_{L}^{(1)}-\frac{1}{\varphi^{L}\left(1+\lambda \hat{B}_{1}^{\prime}(\lambda-\lambda z)\right)}\right]=\frac{1}{1-\rho_{1}} .
$$

Upon substitution of (2.8) and (2.9) into the expressions given in the theorem, the further substitution of (3.8)-(3.10) (as appropriate to the value of $\rho_{1}$ ) into the new expressions completes the proof. 


\section{Further asymptotic analysis of $p_{1}$ and $p_{2}$}

Let us first discuss the statements of Theorem 3.1. Under the assumption that $\rho_{1}<1$, we have (3.2) and (3.3). The probability $p_{1}$ is positive in the limit, while the probability $p_{2}$ vanishes. Under the assumption that $\rho_{1}>1$, we have (3.6) and (3.7). According to these relations, the probability $p_{1}$ vanishes while $p_{2}$ is positive in the limit. This means that if both $J_{1}$ and $J_{2}$ take large, positive values proportional to $L$, then so too will the functional $J$. Specifically, in the case in which $\rho_{1}<1$ we have $J \approx\left(1-\rho_{1}\right) J_{1}$, and in the case $\rho_{1}>1$ we have $J \approx\left[\rho_{2}\left(\rho_{1}-1\right) /\left(\rho_{1}-\rho_{2}\right)\right] J_{2}$.

In the case in which $\rho_{1}=1$, both $p_{1}$ and $p_{2}$ vanish at the rate $L^{-1}$, and $J$ therefore converges to the limit as $L \rightarrow \infty$. Thus, $\rho_{1}=1$ is a possible solution to the control problem, while the cases $\rho_{1}<1$ and $\rho_{1}>1$ are irrelevant. Specifically, for $J \equiv J(L)$ we obtain the following:

$$
\lim _{L \rightarrow \infty} J(L)=j_{1} \frac{\rho_{1,2}}{2}+j_{2} \frac{\rho_{2}}{1-\rho_{2}} \frac{\rho_{1,2}}{2} .
$$

In order now to find the optimal solution, consider the following two cases, in both of which $\delta \rightarrow 0$ as $L \rightarrow \infty$ : (i) $\rho_{1}=1+\delta$; (ii) $\rho_{1}=1-\delta$.

In case (i) we have the following two theorems.

Theorem 4.1. Assume that $\rho_{1}=1+\delta, \delta>0$, and that $L \delta \rightarrow C>0$ as $\delta \rightarrow 0$ and $L \rightarrow \infty$. Assume that $\rho_{1,3} \equiv \rho_{1,3}(\delta)$ is a bounded function of the parameter $\delta$ for $0 \leq \delta<1$, and that the limit $\tilde{\rho}_{1,2}=\lim _{\delta \rightarrow 0} \rho_{1,2}(\delta)$ exists. Then

$$
\begin{aligned}
& p_{1}=\frac{\delta}{\mathrm{e}^{2 C / \tilde{\rho}_{1,2}-1}+o(\delta),} \\
& p_{2}=\frac{\delta \rho_{2} \mathrm{e}^{2 C / \tilde{\rho}_{1,2}}}{\left(1-\rho_{2}\right)\left(\mathrm{e}^{\left.2 C / \tilde{\rho}_{1,2}-1\right)}+o(\delta) .\right.} .
\end{aligned}
$$

Proof. The proof of this theorem is similar to that of [5, Theorem 3.4] and [6, Theorem 4.4]. Under the conditions of the theorem, the following expansion was shown in [18, p. 326]:

$$
\varphi=1-\frac{2 \delta}{\tilde{\rho}_{1,2}}+O\left(\delta^{2}\right) .
$$

Then, by virtue of (4.4), after some algebra we have

$$
1+\lambda \hat{B}_{1}^{\prime}(\lambda-\lambda \varphi)=\delta+O\left(\delta^{2}\right)
$$

Substituting (4.4) and (4.5) into (3.10) yields

$$
\mathrm{E} v_{L}^{(1)}=\frac{\mathrm{e}^{2 C / \tilde{\rho}_{1,2}}-1}{\delta}+O(1),
$$

and from (4.6), (2.8), and (2.9) we obtain the statement of the theorem.

Theorem 4.2. Under the conditions of Theorem 4.1, assume that $C=0$. Then

$$
\begin{aligned}
& \lim _{L \rightarrow \infty} L p_{1}(L)=\frac{\rho_{1,2}}{2}, \\
& \lim _{L \rightarrow \infty} L p_{2}(L)=\frac{\rho_{2}}{1-\rho_{2}} \frac{\rho_{1,2}}{2} .
\end{aligned}
$$


Proof. The statement of the theorem follows by expanding the main terms of the asymptotic relations (4.2) and (4.3) for small $C$.

Notice that (4.7) and (4.8) respectively coincide with (3.4) and (3.5).

In case (ii) we have the following.

Theorem 4.3. Assume that $\rho_{1}=1-\delta, \delta>0$, and that $L \delta \rightarrow C>0$ as $\delta \rightarrow 0$ and $L \rightarrow \infty$. Assume that $\rho_{1,3} \equiv \rho_{1,3}(\delta)$ is a bounded function of the parameter $\delta$ for $0 \leq \delta<1$, and that the limit $\tilde{\rho}_{1,2}=\lim _{\delta \rightarrow 0} \rho_{1,2}(\delta)$ exists. Then

$$
\begin{aligned}
& p_{1}=\delta \mathrm{e}^{\tilde{\rho}_{1,2} / 2 C}+o(\delta), \\
& p_{2}=\delta \frac{\rho_{2}}{1-\rho_{2}}\left(\mathrm{e}^{\tilde{\rho}_{1,2} / 2 C}-1\right)+o(\delta) .
\end{aligned}
$$

Proof. From (3.1) we have

$$
\sum_{n=0}^{\infty} \mathrm{E} v_{n}^{(1)} z^{n}=\frac{\hat{B}_{1}(\lambda-\lambda z)}{\hat{B}_{1}(\lambda-\lambda z)-z} .
$$

The sequence $\left\{\mathrm{E} v_{n}^{(1)}\right\}$ is increasing, and, for $\rho_{1}=1$, from the Tauberian theorem of Hardy and Littlewood (see, e.g. [16], [18], [19], and [20, p. 203]) we obtain

$$
\lim _{L \rightarrow \infty} \frac{E v_{L}^{(1)}}{L}=\lim _{z \uparrow 1}(1-z)^{2} \frac{\hat{B}_{1}(\lambda-\lambda z)}{\hat{B}_{1}(\lambda-\lambda z)-z} .
$$

(It is not difficult to check that (3.9) then follows.) Thus, in the case in which $\rho_{1}=1-\delta$ and $L \delta \rightarrow C$ as $L \rightarrow \infty$, according to the same Tauberian theorem of Hardy and Littlewood, the asymptotic behaviour of $\mathrm{E} v_{L}^{(1)}$ can be found from the asymptotic expansion of

$$
(1-z) \frac{\hat{B}_{1}(\lambda-\lambda z)}{\hat{B}_{1}(\lambda-\lambda z)-z},
$$

as $z \uparrow 1$.

From the Taylor expansion of the denominator of (4.11), we obtain

$$
\begin{aligned}
\frac{1-z}{\hat{B}_{1}(\lambda-\lambda z)-z} & \asymp \frac{1-z}{1-z-\rho_{1}(1-z)+\left(\tilde{\rho}_{1,2} / 2\right)(1-z)^{2}+O\left((1-z)^{3}\right)} \\
& \asymp \frac{1}{\delta+\left(\tilde{\rho}_{1,2} / 2\right)(1-z)+O\left((1-z)^{2}\right)} \\
& \asymp \frac{1}{\delta\left[1+\left(\tilde{\rho}_{1,2} / 2 \delta\right)(1-z)+O\left((1-z)^{2}\right)\right]} \\
& \asymp \frac{1}{\delta \exp \left(\left(\tilde{\rho}_{1,2} / 2 \delta\right)(1-z)\right)}(1+o(1)) .
\end{aligned}
$$

Therefore, assuming that $z=(L-1) / L \rightarrow 1$ as $L \rightarrow \infty$, from (4.12) we obtain the asymptotic behaviour of $\mathrm{E} v_{L}^{(1)}$ as $L \rightarrow \infty$. We have

$$
\mathrm{E} v_{L}^{(1)}=\frac{1}{\delta \mathrm{e}^{\tilde{\rho}_{1,2} / 2 C}}(1+o(1)) .
$$

By now substituting (4.13) into (2.8) and (2.9), we obtain the desired statements of the theorem. 
Theorem 4.4. Under the conditions of Theorem 4.3, assume that $C=0$. Then we obtain (4.7) and (4.8).

Proof. The statement of the theorem follows by expanding the main terms of the asymptotic relations (4.9) and (4.10) for small $C$.

\section{Solution of the control problem}

In this section we formulate the theorem characterizing the solution to the control problem. For $J \equiv J(L)$ we have the following limiting relation:

$$
\begin{aligned}
\lim _{L \rightarrow \infty} J(L) & =\lim _{L \rightarrow \infty}\left[p_{1}(L) J_{1}(L)+p_{2}(L) J_{2}(L)\right] \\
& =j_{1} \lim _{L \rightarrow \infty} L p_{1}(L)+j_{2} \lim _{L \rightarrow \infty} L p_{2}(L) .
\end{aligned}
$$

By substituting (4.2) and (4.3) into the right-hand side of (5.1) and taking into account the fact that $L \delta \rightarrow C$, we obtain

$$
\begin{aligned}
& J^{\text {upper }}=\lim _{L \rightarrow \infty} J(L) \\
& =\left[j_{1} \frac{1}{\mathrm{e}^{2 C / \tilde{\rho}_{1,2}}-1}+j_{2} \frac{\rho_{2} \mathrm{e}^{2 C / \tilde{\rho}_{1,2}}}{\left(1-\rho_{2}\right)\left(\mathrm{e}^{2 C / \tilde{\rho}_{1,2}}-1\right)}\right] \lim _{L \rightarrow \infty} L \delta \\
& =C\left[j_{1} \frac{1}{\mathrm{e}^{2 C / \tilde{\rho}_{1,2}}-1}+j_{2} \frac{\rho_{2} \mathrm{e}^{2 C / \tilde{\rho}_{1,2}}}{\left(1-\rho_{2}\right)\left(\mathrm{e}^{2 C / \tilde{\rho}_{1,2}}-1\right)}\right] \text {. }
\end{aligned}
$$

By substituting (4.9) and (4.10) into the right-hand side of (5.1) and taking into account the fact that $L \delta \rightarrow C$, we in turn obtain

$$
J^{\text {lower }}=C\left[j_{1} \mathrm{e}^{\tilde{\rho}_{1,2} / 2 C}+j_{2} \frac{\rho_{2}}{1-\rho_{2}}\left(\mathrm{e}^{\tilde{\rho}_{1,2} / 2 C}-1\right)\right] .
$$

Let us now study the functionals $J^{\text {upper }}$ and $J^{\text {lower }}$ given by (5.2) and (5.3). Notice that (5.2) involves the constants $j_{1}, j_{2}$, and $\rho_{2}$. Let us assume that these constants are such that

$$
j_{1}=j_{2} \frac{\rho_{2}}{1-\rho_{2}} .
$$

Then $C=0$ is the parameter value minimizing the functional $J^{\text {upper }}$. Indeed, in this case

$$
\begin{aligned}
J^{\text {upper }} & =j_{1} C\left[\frac{1}{\mathrm{e}^{2 C / \tilde{\rho}_{1,2}}-1}+\frac{\mathrm{e}^{2 C / \tilde{\rho}_{1,2}}}{\mathrm{e}^{2 C / \tilde{\rho}_{1,2}}-1}\right] \\
& =j_{1} C\left[\frac{1}{\mathrm{e}^{2 C / \tilde{\rho}_{1,2}}-1}+\frac{\left(\mathrm{e}^{2 C / \tilde{\rho}_{1,2}}-1\right)+1}{\mathrm{e}^{2 C / \tilde{\rho}_{1,2}}-1}\right] \\
& =j_{1} C\left[1+\frac{2}{\mathrm{e}^{2 C / \tilde{\rho}_{1,2}}-1}\right] .
\end{aligned}
$$

Therefore, we have $\lim _{C \rightarrow 0} J^{\text {upper }}=j_{1} \tilde{\rho}_{1,2}$, and to the right of the point $C=0$ the function $J^{\text {upper }}$ is increasing in $C$. Hence, (5.4) is the condition for $C=0$ to minimise $J^{\text {upper }}$.

Next, consider

$$
\frac{\mathrm{d}}{\mathrm{d} C}\left[\frac{C}{\mathrm{e}^{2 C / \tilde{\rho}_{1,2}}-1}\right]=\frac{\mathrm{e}^{2 C / \tilde{\rho}_{1,2}}-1-\left(2 C^{2} / \tilde{\rho}_{1,2}\right) \mathrm{e}^{2 C / \tilde{\rho}_{1,2}}}{\left(\mathrm{e}^{2 C / \tilde{\rho}_{1,2}}-1\right)^{2}}
$$


and

$$
\frac{\mathrm{d}}{\mathrm{d} C}\left[\frac{C \mathrm{e}^{2 C / \tilde{\rho}_{1,2}}}{\mathrm{e}^{2 C / \tilde{\rho}_{1,2}}-1}\right]=\frac{\mathrm{d}}{\mathrm{d} C}\left[\frac{C}{\mathrm{e}^{2 C / \tilde{\rho}_{1,2}}-1}\right] \mathrm{e}^{2 C / \tilde{\rho}_{1,2}}+\frac{2}{\tilde{\rho}_{1,2}}\left[\frac{C}{\mathrm{e}^{2 C / \tilde{\rho}_{1,2}}-1}\right] \mathrm{e}^{2 C / \tilde{\rho}_{1,2}} .
$$

Clearly (5.6) is not smaller than (5.5), and they are equal when $C=0$. Therefore, if the right-hand side of (5.4) is greater than the left-hand side of (5.4), then $C=0$ remains the value minimizing the functional $J^{\text {upper }}$. A similar result holds for the functional $J^{\text {lower }}$ given in (5.3). Specifically, if the right-hand side of (5.4) is not greater than the left-hand side of (5.4), then $C=0$ is the value minimizing the functional $J^{\text {lower }}$.

Thus, the solution to the control problem is given in the following theorem.

Theorem 5.1. If the parameters $\lambda$ and $\rho_{2}$ are given, then the optimal solution to the control problem is as follows.

- If

$$
j_{1}=\frac{\rho_{2}}{1-\rho_{2}} j_{2}
$$

then the optimal solution to the control problem is achieved for $\rho_{1}=1$.

- If

$$
j_{1}>\frac{\rho_{2}}{1-\rho_{2}} j_{2}
$$

then the optimal solution to the control problem is a minimization of the functional $J^{\text {upper }}$. The optimal solution is achieved for $\rho_{1}=1+\delta$, where $\delta(L)$ is a small, positive parameter and $L \delta(L) \rightarrow C$, the nonnegative parameter minimizing (5.2).

- If

$$
j_{1}<\frac{\rho_{2}}{1-\rho_{2}} j_{2}
$$

then the optimal solution to the control problem is a minimization of the functional $J^{\text {lower }}$. The optimal solution is achieved for $\rho_{1}=1-\delta$, where $\delta(L)$ is a small, positive parameter and $L \delta(L) \rightarrow C$, the nonnegative parameter minimizing (5.3).

\section{Concluding remarks}

In this paper we have posed and solved a control problem for a large dam. The main specification of the problem is that the performance criteria take into account passage across the lower and upper levels. The solution to the control problem is asymptotically independent of the explicit form of the probability distribution functions $B_{1}(x)$ and $B_{2}(x)$, and, under the assumption that the parameters $\lambda$ and $\rho_{2}$ are given, depending on the performance criteria the parameter $\rho_{1}$ must take the form $\rho_{1}=1, \rho_{1}=1+\delta(L)$, or $\rho_{1}=1-\delta(L)$, where $\delta(L)>0$ and, as $L \rightarrow \infty, \delta(L)$ vanishes and $L \delta(L) \rightarrow C$.

\section{Acknowledgement}

This research was supported by the Australian Research Council, grant no. DP0771338. 


\section{References}

[1] Abdel-Hameed, M. (2000). Optimal control of a dam using $P_{\lambda, \tau}^{M}$ policies and penalty cost when the input process is a compound Poisson process with positive drift. J. Appl. Prob. 37, 408-416.

[2] Abdel-Hameed, M. and NaKhi, Y. (1990). Optimal control of a finite dam using $P_{\lambda, \tau}^{M}$ policies and penalty cost: total discounted and long-run average cases. J. Appl. Prob. 27, 888-898.

[3] Aвramov, V. M. (1991). Investigation of a Queueing System with Service Depending on a Queue-Length. Donish, Dushanbe, Tadzhikistan (in Russian).

[4] Abramov, V. M. (1997). On a property of a refusals stream. J. Appl. Prob. 37, 800-805.

[5] Abramov, V. M. (2002). Asymptotic analysis of the GI/M/1/n queueing system as $n$ increases to infinity. Ann. Operat. Res. 112, 35-41.

[6] Abramov, V. M. (2004). Asymptotic behavior of the number of lost messages. SIAM J. Appl. Math. 64, 746-761.

[7] Bae, J., KIM, S. and LeE, E. Y. (2002). A $P_{\lambda}^{M}$ policy for an M/G/1 queueing system. Appl. Math. Modelling 26, 929-939.

[8] Bae, J., Kim, S. And LeE, E. Y. (2003). Average cost under the $P_{\lambda, \tau}^{M}$ policy in a finite dam with compound Poisson inputs. J. Appl. Prob. 40, 519-526.

[9] Boxma, O., Kaspi, H., Kella, O. and Perry, D. (2005). On/off storage systems with state-dependent input, output, and switching rates. Prob. Eng. Inf. Sci. 19, 1-14.

[10] Faddy, M. J. (1974). Optimal control of finite dams: discrete (2-stage) output procedure. J. Appl. Prob. 11, $111-121$

[11] Feller, W. (1971). An Introduction to Probability Theory and Its Applications, Vol. 2. Wiley, New York.

[12] Kaspi, H., Kella, O. AND Perry, D. (1996). Dam processes with state-dependent batch sizes, and intermittent production processes with state-dependent rates. Queueing Systems 24, 37-57.

[13] Lam, Y. and Lou, J. H. (1987). Optimal control for a finite dam. J. Appl. Prob. 24, 196-199.

[14] Lee, E. Y. AND AhN, S. K. (1998). $P_{\tau}^{M}$ policy for a dam with input formed by a compound Poisson process. $J$. Appl. Prob. 35, 482-488.

[15] Phatarfod, R. M. (1989). Riverflow and reservoir storage models. Math. Comput. Modelling 12, 1057-1077.

[16] Postnikov, A. G. (1979). Tauberian theory and its applications. Trudy Mat. Inst. Steklov 144, 147 pp. (in Russian). English translation: Proc. Steklov Inst. Math. 1980, 138 pp.

[17] Ross, S. M. (1983). Stochastic Processes. John Wiley, New York.

[18] Subhankulov, M. A. (1976). Tauberian Theorems with Remainder. Nauka, Moscow (in Russian).

[19] Sznajder, R. AND Filar, J. A. (1992). Some comments on a theorem of Hardy and Littlewood. J. Optimization Theory Appl. 75, 201-208.

[20] TAKács, L. (1967). Combinatorial Methods in the Theory of Stochastic Processes. John Wiley, New York.

[21] Zukerman, D. (1977). Two-stage output procedure of a finite dam. J. Appl. Prob. 14, 421-425. 